\title{
LOWER AND UPPER REGULARIZATIONS OF FRAME SEMICONTINUOUS REAL FUNCTIONS
}

\author{
JAVIER GUTIÉRREZ GARCÍA, TOMASZ KUBIAK AND JORGE PICADO
}

\begin{abstract}
As discovered recently, Li and Wang's 1997 treatment of semicontinuity for frames does not faithfully reflect the classical concept. In this paper we continue our study of semicontinuity in the pointfree setting. We define the pointfree concepts of lower and upper regularizations of frame semicontinuous real functions. We present characterizations of extremally disconnected frames in terms of these regularizations that allow us to reprove, in particular, the insertion and extension type characterizations of extremally disconnected frames due to Y.-M. Li and Z.-H. $\mathrm{Li}$ [Algebra Universalis 44 (2000), 271-281] in the right semicontinuity context. It turns out that the proof of the insertion theorem becomes very easy after having established a number of basic results regarding the regularizations. Notably, our extension theorem is a much strengthened version of Li and Li's result and it is proved without making use of the insertion theorem.
\end{abstract}

KEYWORDS: Frame, locale, sublocale, frame of reals, lower and upper frames of reals, frame semicontinuous real function, lower and upper semicontinuous real functions, lower and upper regularizations, extremal disconnectedness, insertion theorem, extension theorem.

AMS Subject Classification (2000): 06D22, 54C30, 54G05.

\section{Introduction}

Ten years ago, Li and Wang [13] introduced pointfree lower and upper semicontinuities for morphisms from certain frames of reals into arbitrary frames. However, their concepts do not faithfully reflect the classical concepts of semicontinuity for real-valued functions. Indeed, it was shown in [14] that the insertion theorem of Li and Wang, as stated in [13], is not correct in the sense that it fails to capture the classical Katětov-Tong insertion theorem due to the inappropriateness of the pointfree semicontinuities they introduced (see [14, Example 4.2]). The discrepancy between their concepts and the usual

Received September 10, 2007.

The first and the second named authors acknowledge financial support from the Ministry of Education and Science of Spain and FEDER under grant MTM2006-14925-C02-02. The first named author also acknowledges financial support from the University of the Basque Country under grant UPV05/101. The third named author acknowledges financial support from the Centre of Mathematics of the University of Coimbra/FCT. 
topological ones has been fixed by the first and third named authors in [7] who determined the concepts of pointfree semicontinuities that avoid the shortcomings of the ones in [13].

With the semicontinuity notions of [13], $\mathrm{Li}$ and $\mathrm{Li}$ [12] went to characterize extremally disconnected frames in terms of insertion and extension theorems. The main scope of this paper is to reprove their characterizations in the framework of the semicontinuities in the sense of [7]. In doing so we first define a suitable notion of regularization of semicontinuous frame homomorphisms. With this concept at hand, we can proceed similarly as in the topological case developed in [10] and provide a true generalization of the insertion theorem for extremally disconnected frames. Even if the semicontinuities used in [12] continue to be incorrect, the insertion theorem of [12] can be saved. This time (unlike the situation with the insertion theorem of [13]) it is so due to the nature of the functions involved in the insertion theorem. More precisely, we make the important observation that if a lower semicontinuous map minorizes an upper semicontinuous one (both in the sense of [13] and [12]), then this circumstance necessitates both the maps to be semicontinuous in the right sense of [7] which is being used in this paper. This observation renders the two insertion theorems (of [12] and of this paper) equivalent. We also provide an extension theorem for extremally disconnected frames that strengthens the version in [12] and that, unlike [12], is obtained by a direct argument that avoids reference to the insertion theorem.

\section{Background on frames}

Frames and locales. Pointfree topology deals with the category Frm of frames and its dual, the category Loc of locales. The objects of the category Frm are complete lattices $L$ (with 1 and 0 as top and bottom, respectively) in which

$$
a \wedge \bigvee B=\bigvee\{a \wedge b: b \in B\}
$$

for all $a \in L$ and $B \subseteq L$. Morphisms of Frm, called frame homomorphisms, are maps between frames which preserve arbitrary joins (hence the top) and finite meets (hence the bottom). The set of all morphisms from $L$ into $M$ is denoted by $\operatorname{Frm}(L, M)$. One source of frames is given by the lattice $\mathcal{O} X$ of all open subsets of a topological space $X$. The assignment $X \mapsto \mathcal{O} X$ gives rise to a contravariant functor $\mathcal{O}:$ Top $\rightarrow$ Frm which makes a continuous map $f: X \rightarrow Y$ into the frame homomorphism $\mathcal{O} f: \mathcal{O} Y \rightarrow \mathcal{O} X$ determined by $\mathcal{O} f(U)=f^{-1}(U)$ for all $U \in \mathcal{O} Y$. 
Heyting operator. If $L$ is a frame, then $a \wedge(\cdot): L \rightarrow L$ is $\bigvee$-preserving and, thus, has a right adjoint $a \rightarrow(\cdot): L \rightarrow L$ satisfying

$$
a \wedge c \leq b \text { iff } c \leq a \rightarrow b .
$$

Then

$$
a \rightarrow b=\bigvee\{c \in L: a \wedge c \leq b\}
$$

The map $x \mapsto(a \rightarrow x)$ is order-preserving and the map $x \mapsto(x \rightarrow a)$ is order-reversing. In particular:

$(\mathrm{H} 1) a \wedge(a \rightarrow b)=a \wedge b$,

(H2) $(\bigvee A) \rightarrow b=\bigwedge_{a \in A}(a \rightarrow b)$,

for $a, b \in L$ and $A \subseteq L$. The pseudocomplement of $a \in L$ is $a^{*}=a \rightarrow 0$. Then $a \wedge a^{*}=0, a \leq a^{* *}$ and $(\bigvee A)^{*}=\bigwedge_{a \in A} a^{*}$. Also, $a \leq b$ implies $b^{*} \leq a^{*}$. In particular, $a^{* * *}=a$.

Sublocales. The quotients in Frm (equivalently, the subobjects in Loc) are called sublocales (see [8, page 50] and [15]). There are several equivalent ways of describing them (cf. [15] or [16]). Here we use the approach of [15]: an $S \subseteq L$ is a sublocale of $L$ if, given $A \subseteq S$ and $a \in L$, one has $\bigwedge A \in S$ and $a \rightarrow s \in S$ for all $s \in S$. It determines the surjection (frame quotient) $c_{S}: L \rightarrow S$ given by

$$
c_{S}(x)=\bigwedge\{s \in S: x \leq s\} .
$$

Each sublocale $S \subseteq L$ is a frame with the same meets as in $L$ and with the same Heyting operation $\rightarrow$. In general, the bottom $0_{S}$ of $S$ may differ from 0 , but 1 is always the top in $S$. The sublocales of $L$ form a complete lattice under inclusion in which $\{1\}$ is the bottom. For any $a \in L$, the set

$$
\mathfrak{o}(a)=\{a \rightarrow b: b \in L\}=\{b \in L: a \rightarrow b=b\}
$$

is a sublocale of $L$ and $0_{\mathfrak{o}(a)}=a^{*}$. It will be referred as an open sublocale of $L$. Its corresponding frame quotient $c_{\mathfrak{o}(a)}: L \rightarrow \mathfrak{o}(a)$ is given by

$$
c_{\mathfrak{o}(a)}(x)=a \rightarrow x .
$$

Remarks 2.1. (1) The sublocale $\mathfrak{o}(a)$ viewed as a quotient of $L$ is isomorphic to the quotient $\downarrow a$ (with the quotient map $x \mapsto a \wedge x$ ). The isomorphism from $\mathfrak{o}(a)$ to $\downarrow a$ is given by $(a \rightarrow b) \mapsto a \wedge(a \rightarrow b)=a \wedge b$.

(2) In $S=\mathfrak{o}\left(a \vee a^{*}\right)$, the bottom $0_{S}=\left(a \vee a^{*}\right)^{*}=a^{*} \wedge a^{* *}=0$. Also, $a^{*}$ and $a^{*} \rightarrow a$ belong to $S$ and are complements to each other. Indeed, by (H2),

$$
c_{S}\left(a^{*}\right)=\left(a \vee a^{*}\right) \rightarrow a^{*}=a^{*} \in S
$$


and

$$
c_{S}(a)=\left(a \vee a^{*}\right) \rightarrow a=a^{*} \rightarrow a \in S .
$$

Thus $a^{*} \wedge\left(a^{*} \rightarrow a\right)=a^{*} \wedge a=0$ and

$$
a^{*} \vee_{S}\left(a^{*} \rightarrow a\right)=c_{S}\left(a^{*}\right) \vee_{S} c_{S}(a)=c_{S}\left(a^{*} \vee a\right)=1
$$

Lemma 2.2. If $A \subseteq L$ and $\bigvee_{a \in A} a^{*}=1$, then $\bigcap_{a \in A} \mathfrak{o}(a)=\{1\}$.

Proof: If $x \in \bigcap_{a \in A} \mathfrak{o}(a)$, then $x=a \rightarrow x \geq a \rightarrow 0=a^{*}$ for all $a \in A$. Thus $x \geq \bigvee_{a \in A} a^{*}=1$.

We will show later on, in a more specific context, that the reverse implication of Lemma 2.2 does not hold in general (see Example 3.10).

Other concepts will be recalled when actually needed. For more information on frames and locales we refer to [8] and [16].

Note. Up to Section $5, L$ stands for an arbitrary frame.

\section{Semicontinuity of real functions on frames}

Recall that $A \subseteq L$ generates $L$ if each element of $L$ is a join of a family of meets of finite subsets of $A$. The category Frm being algebraic allows definitions by generators and relations. In particular, one may define the frame of reals in terms of the rationals in a constructive way:

Definition 3.1 ([1] and [2]). The frame of reals is the frame $\mathfrak{L}(\mathbb{R})$ generated by all ordered pairs $(p, q)$, where $p, q \in \mathbb{Q}$, subject to the following relations:

$(\mathrm{R} 1)(p, q) \wedge(r, s)=(p \vee r, q \wedge s)$,

(R2) $(p, q) \vee(r, s)=(p, s)$ whenever $p \leq r<q \leq s$,

(R3) $(p, q)=\bigvee\{(r, s): p<r<s<q\}$,

(R4) $1=\bigvee_{p, q \in \mathbb{Q}}(p, q)$.

We write: $(p,-)=\bigvee_{q \in \mathbb{Q}}(p, q)$ and $(-, q)=\bigvee_{p \in \mathbb{Q}}(p, q)$.

Members of $\mathrm{C}(L)=\operatorname{Frm}(\mathfrak{L}(\mathbb{R}), L)$ are called continuous real functions $[1]$ on $L$.

Remark 3.2. Viewing $(r,-)$ and $(-, r)$ as primitive notions, the frame $\mathfrak{L}(\mathbb{R})$ can equivalently be described as the frame having generators of the form $(r,-)$ and $(-, r)$ subject to the following relations (cf. [13]): 
$\left(\mathrm{R} 1^{\prime}\right)(-, r) \vee(s,-)=1$, whenever $s<r$,

$\left(\mathrm{R} 2^{\prime}\right)(-, r) \wedge(s,-)=0$, whenever $r \leq s$,

$\left(\mathrm{R} 3^{\prime}\right)(r,-)=\bigvee_{s>r}(s,-)$,

$\left(\mathrm{R} 4^{\prime}\right)(-, r)=\bigvee_{s<r}(-, s)$,

$\left(\mathrm{R} 5^{\prime}\right) \bigvee_{r \in \mathbb{Q}}(r,-)=1$,

$\left(\mathrm{R} 6^{\prime}\right) \bigvee_{r \in \mathbb{Q}}(-, r)=1$.

With $(p, q)=(p,-) \wedge(-, q)(p<q)$ one goes back to (R1)-(R4).

The subframes of $\mathfrak{L}(\mathbb{R})$ generated by the elements $(r,-)$ satisfying $\left(\mathrm{R} 3^{\prime}\right)$ and $\left(\mathrm{R} 5^{\prime}\right)$ (resp., $(-, r)$ satisfying $\left(\mathrm{R} 4^{\prime}\right)$ and $\left(\mathrm{R} 6^{\prime}\right)$ ) will be, respectively, denoted by

$$
\mathfrak{L}_{u}(\mathbb{R}) \quad \text { and } \quad \mathfrak{L}_{l}(\mathbb{R}) .
$$

The study of semicontinuity for frames was undertaken by Li and Wang [13] with the families

$$
\operatorname{Frm}\left(\mathfrak{L}_{u}(\mathbb{R}), L\right) \quad \text { and } \quad \operatorname{Frm}\left(\mathfrak{L}_{l}(\mathbb{R}), L\right) .
$$

For why those two families are not the right pointfree counterparts of the sets $\operatorname{LSC}(X, \mathbb{R})$ and $\operatorname{USC}(X, \mathbb{R})$ of all lower and upper semicontinuous real functions on a topological space $X$ we refer to [7, Corollary 4.3]. Before recalling the adequate analogs of $[7]$ we first state some properties of $\operatorname{Frm}\left(\mathfrak{L}_{u}(\mathbb{R}), L\right)$ and $\operatorname{Frm}\left(\mathfrak{L}_{l}(\mathbb{R}), L\right)$.

Remarks 3.3. (1) With the pointwise partial order on $\operatorname{Frm}\left(\mathfrak{L}_{u}(\mathbb{R}), L\right)$ :

$$
f_{1} \leq f_{2} \quad \Leftrightarrow \quad f_{1}(r,-) \leq f_{2}(r,-) \quad \text { for all } r \in \mathbb{Q},
$$

$\operatorname{Frm}\left(\mathfrak{L}_{u}(\mathbb{R}), L\right)$ is closed under arbitrary nonempty joins and finite meets which are pointwise too. E.g., if $\varnothing \neq \mathcal{F} \subseteq \operatorname{Frm}\left(\mathfrak{L}_{u}(\mathbb{R}), L\right)$, then

$$
(\bigvee \mathcal{F})(r,-)=\bigvee_{f \in \mathcal{F}} f(r,-)
$$

The constant map with value 1 is the top element, while there is no bottom element in $\operatorname{Frm}\left(\mathfrak{L}_{u}(\mathbb{R}), L\right)$.

(2) $\operatorname{Frm}\left(\mathfrak{L}_{l}(\mathbb{R}), L\right)$ is partially ordered by the reverse pointwise ordering:

$$
g_{1} \leq g_{2} \quad \Leftrightarrow \quad g_{2}(-, r) \leq g_{1}(-, r) \quad \text { for all } r \in \mathbb{Q},
$$

under which it is closed with respect to arbitrary nonempty meets and finite joins. These operations are pointwise with respect to the reverse ordering. 
For example, given $\varnothing \neq \mathcal{G} \subseteq \operatorname{Frm}\left(\mathfrak{L}_{l}(\mathbb{R}), L\right)$ one has

$$
(\bigwedge \mathcal{G})(-, r)=\bigvee_{g \in \mathcal{G}} g(-, r) \text {. }
$$

The constant map with value 1 is the bottom element, while there is no top element in $\operatorname{Frm}\left(\mathfrak{L}_{l}(\mathbb{R}), L\right)$.

(3) A saving of effort will be achieved by making use of the isomorphism

$$
-(\cdot): \operatorname{Frm}\left(\mathfrak{L}_{u}(\mathbb{R}), L\right) \rightarrow \operatorname{Frm}\left(\mathfrak{L}_{l}(\mathbb{R}), L\right)
$$

defined by

$$
(-f)(-, r)=f(-r,-) \quad \text { for all } r \in \mathbb{Q} .
$$

Remark 3.4. A morphism having $\mathfrak{L}_{u}(\mathbb{R})$ or $\mathfrak{L}_{l}(\mathbb{R})$ as a domain will be defined on their generators. Such a map uniquely determines a frame homomorphism if and only if it makes the relations holding for generators into identities on the codomain frame. For example, $(r,-) \mapsto f(r,-)$ determines a frame homomorphism $f: \mathfrak{L}_{u}(\mathbb{R}) \rightarrow L$ if and only if $f(r,-)=\bigvee_{s>r} f(s,-)$ and $\bigvee_{r \in \mathbb{Q}} f(r,-)=1$ hold true $\left(\right.$ cf. $\left(\mathrm{R} 3^{\prime}\right)$ and $\left.\left(\mathrm{R} 5^{\prime}\right)\right)$.

The following relations of minorization and majorization (see [14]) generalize the usual pointwise way of comparing members of $\operatorname{LSC}(X, \mathbb{R})$ and $\operatorname{USC}(X, \mathbb{R})$ by overcoming the fact that members of $\operatorname{Frm}\left(\mathfrak{L}_{u}(\mathbb{R}), L\right)$ and $\operatorname{Frm}\left(\mathfrak{L}_{l}(\mathbb{R}), L\right)$ have distinct domains.

Definition 3.5. Let $f \in \operatorname{Frm}\left(\mathfrak{L}_{u}(\mathbb{R}), L\right)$ and $g \in \operatorname{Frm}\left(\mathfrak{L}_{l}(\mathbb{R}), L\right)$. Then:

(1) $f$ is said to minorize $g$ (written: $f \triangleleft g$ ) iff

$$
f(r,-) \wedge g(-, r)=0 \quad \text { for all } r \text { in } \mathbb{Q} .
$$

(2) $f$ is said to majorize $g$ (written: $f \triangleright g$ ) iff

$$
f(r,-) \vee g(-, s)=1 \quad \text { for all } r<s \text { in } \mathbb{Q} .
$$

Thus $f \triangleleft g$ iff $f(r,-) \leq g(-, r)^{*}$ for all $r \in \mathbb{Q}$ (we write $g(-, r)^{*}$ rather than $\left.(g(-, r))^{*}\right)$. Also $f \triangleleft g$ iff $-g \triangleleft-f$, and $f \triangleright g$ iff $-g \triangleright-f$.

We eventually quote from [7] the definition of lower and upper semicontinuities in pointfree topology. We note that the original formulation has been phrased in [7] in terms of congruences. It will be stated here in terms of sublocales. 
Definition 3.6 ([7]). (1) A lower semicontinuous real function (l.s.c. for short) on $L$ is a morphism $f: \mathfrak{L}_{u}(\mathbb{R}) \rightarrow L$ such that

$$
\bigcap_{r \in \mathbb{Q}} \mathfrak{o}(f(r,-))=\{1\} \text {. }
$$

(2) An upper semicontinuous real function (u.s.c. for short) on $L$ is a morphism $g: \mathfrak{L}_{l}(\mathbb{R}) \rightarrow L$ such that

$$
\bigcap_{r \in \mathbb{Q}} \mathfrak{o}(g(-, r))=\{1\} .
$$

The collections of all l.s.c. and u.s.c. real functions on $L$ are denoted by $\operatorname{LSC}(L)$ and $\operatorname{USC}(L)$, respectively. Clearly, $f$ is l.s.c. if and only if $-f$ is u.s.c..

For more information on lower and upper semicontinuous real functions on frames see [7] and [4].

Notation. Given $g \in \operatorname{USC}(L)$ and $f \in \operatorname{LSC}(L)$, we put

$$
\downarrow_{\mathrm{LSC}}(g)=\{f \in \operatorname{LSC}(L): f \triangleleft g\} \quad \text { and } \quad \uparrow^{\mathrm{USC}}(f)=\{g \in \operatorname{USC}(L): f \triangleleft g\} \text {. }
$$

Furthermore, we put

$$
\operatorname{LSC}^{-}(L)=\left\{f \in \operatorname{LSC}(L): \uparrow^{\mathrm{USC}}(f) \neq \varnothing\right\}
$$

and

$$
\mathrm{USC}^{\circ}(L)=\left\{g \in \operatorname{USC}(L): \downarrow_{\mathrm{LSC}}(g) \neq \varnothing\right\} .
$$

We observe that both $\operatorname{LSC}^{-}(L)$ and $\operatorname{USC}^{\circ}(L)$ are lattices under the ordering inherited from $\operatorname{LSC}(L)$ and $\operatorname{USC}(L)$.

Definition 3.7. Members of $\operatorname{LSC}^{-}(L)$ (resp., $\mathrm{USC}^{\circ}(L)$ ) are called proper lower (resp., upper) semicontinuous real functions on $L$.

The following provides a link between the relation of minorization and the algebraic conditions of Definition 3.6.

Lemma 3.8. Let $f,-g \in \operatorname{Frm}\left(\mathfrak{L}_{u}(\mathbb{R}), L\right)$. Then the following hold:

(1) If $f$ minorizes $g$, then $\bigvee_{r \in \mathbb{Q}} f(r,-)^{*}=1=\bigvee_{r \in \mathbb{Q}} g(-, r)^{*}$.

(2) If $f$ minorizes $g$, then $f \in \mathrm{LSC}(L)$ and $g \in \mathrm{USC}(L)$.

(3) $g \in \mathrm{USC}^{\circ}(L)$ if and only if $\bigvee_{r \in \mathbb{Q}} g(-, r)^{*}=1$.

(4) $f \in \mathrm{LSC}^{-}(L)$ if and only if $\bigvee_{r \in \mathbb{Q}} f(r,-)^{*}=1$

(5) $f \in \operatorname{LSC}^{-}(L)$ if and only if $-f \in \operatorname{USC}^{\circ}(L)$. 
Proof: (1) We have $f(r,-) \leq g(-, r)^{*}$ for all $r \in \mathbb{Q}$, hence

$$
1=\bigvee_{r \in \mathbb{Q}} f(r,-) \leq \bigvee_{r \in \mathbb{Q}} g(-, r)^{*} .
$$

The second equality follows from the first, since $-g \triangleleft-f$.

(2) By (1) and Lemma 2.2.

(3) Statement (1) yields the forward implication. For the backward one, assume $\bigvee_{r \in \mathbb{Q}} g(-, r)^{*}=1$. We construct a frame homomorphism $h: \mathfrak{L}_{u}(\mathbb{R}) \rightarrow$ $L$ which minorizes $g$. We put

$$
h(r,-)=\bigvee_{s>r} g(-, s)^{*}
$$

for all $r \in \mathbb{Q}$. This assignment turns conditions $\left(\mathrm{R} 3^{\prime}\right)$ and $\left(\mathrm{R} 5^{\prime}\right)$ into identities on $L$ :

$$
\bigvee_{s>r} h(s,-)=\bigvee_{s>r} \bigvee_{t>s} g(-, t)^{*}=\bigvee_{t>r} g(-, t)^{*}=h(r,-)
$$

and

$$
\bigvee_{r \in \mathbb{Q}} h(r,-)=\bigvee_{r \in \mathbb{Q}} \bigvee_{s>r} g(-, s)^{*}=\bigvee_{r \in \mathbb{Q}} g(-, r)^{*}=1
$$

So, $h \in \operatorname{Frm}\left(\mathfrak{L}_{u}(\mathbb{R}), L\right)$. Clearly,

$$
h(r,-)=\bigvee_{s>r} g(-, s)^{*} \leq g(-, r)^{*},
$$

hence $h \triangleleft g$.

(4) This is dual to (3) via $f \mapsto-f$ of (3) in Remarks 3.3.

(5) This follows immediately from (3) in Remarks 3.3.

Remark 3.9. We have the following chain of proper inclusions

$$
\operatorname{LSC}^{-}(L) \subset \operatorname{LSC}(L) \subset \operatorname{Frm}\left(\mathfrak{L}_{u}(\mathbb{R}), L\right)
$$

and the same for the case of upper semicontinuity. Indeed, in [7] there is a number of examples which show that the second inclusion is proper indeed. A spatial example showing that the first inclusion is proper too is given below.

Example 3.10. Consider $\mathbb{R}_{+}=[0, \infty[$ with the usual topology and a l.s.c. function $f: \mathbb{R}_{+} \rightarrow \mathbb{R}$ given by $f(0)=0$ and $f(x)=1 / x$ if $x>0$. Put $L=\mathcal{O} \mathbb{R}_{+}$and think of $f$ as a morphism $f: \mathfrak{L}_{u}(\mathbb{R}) \rightarrow L$. Then

$$
f(r,-)= \begin{cases}] 0, \frac{1}{r}[ & \text { if } r>0 \\ ] 0, \infty[ & \text { if } r=0 \\ \mathbb{R}_{+} & \text {if } r<0\end{cases}
$$


If $W \in \bigcap_{r \in \mathbb{Q}} \mathfrak{o}(f(r,-))$, then

$$
\left.W=f(r,-) \rightarrow W \supseteq f(r,-)^{*}=\right] \frac{1}{r}, \infty[\text { for all } r>0 .
$$

Thus $] 0, \infty[\subseteq W$. However $] 0, \infty[\neq W$ because $f(0,-) \rightarrow] 0, \infty\left[=\mathbb{R}_{+}\right.$. Thus $W=\mathbb{R}_{+}$, hence

$$
\bigcap_{r \in \mathbb{Q}} \mathfrak{o}(f(r,-))=\left\{\mathbb{R}_{+}\right\}
$$

and $f \in \operatorname{LSC}(L)$. However,

$$
\left.\bigcup_{r \in \mathbb{Q}} f(r,-)^{*}=\right] 0, \infty\left[\neq \mathbb{R}_{+} .\right.
$$

By Lemma $3.8(4)$, the just proved inequality means that $f \notin \operatorname{LSC}^{-}(L)$.

\section{Semicontinuous regularization}

To provide motivation for regularizations of l.s.c. and u.s.c. real functions on frames, let us have a look at the (classical) topological setting.

Discussion. (1) Each (not necessarily continuous) function $h: X \rightarrow \mathbb{R}$ on a topological space $X$ admits a lower regularization $h_{*}: X \rightarrow \overline{\mathbb{R}}$ (extended reals) defined by $h_{*}(x)=\bigvee\{\bigwedge f(U): x \in U \in \mathcal{O} X\}$ for all $x \in X$. Then $h_{*}$ is the biggest lower semicontinuous minorant of $h$, i.e.,

$$
h_{*}=\bigvee\{f \in \operatorname{LSC}(X, \mathbb{R}): f \leq h\} .
$$

Moreover, for each $r \in \mathbb{Q}$ one has

$$
h_{*}^{-1}(] r, \infty[)=\bigcup_{s>r} \operatorname{Int}\left(h ^ { - 1 } \left([s,-\infty[))=\bigcup_{s>r}\left(h^{-1}(]-\infty, s[)\right)^{*}\right.\right.
$$

(notice that in the frame $\mathcal{O} X$ the pseudocomplement $U^{*}$ of an open $U$ is given by $\operatorname{Int}(X \backslash U))$. All this means that $h_{*}$ takes values in $\mathbb{R}$ iff it has a l.s.c. minorant iff $\bigcup_{r \in \mathbb{Q}}\left(h^{-1}(]-\infty, r[)^{*}=X\right.$ (cf. condition (3) of Lemma 3.8). Similar discussion applies to the upper regularization $h^{*}=-(-h)_{*}$.

(2) It follows from the above formula that - in a pointfree setting - one can without trouble produce lower regularizations of members of $\operatorname{USC}^{\circ}(L)$ (and, dually, upper regularizations of members of $\mathrm{LSC}^{-}(L)$ ). So we shall do in what follows. Such a machinery will be sufficient for the main purpose of this paper. 
Lemma 4.1. Let $g \in \mathrm{USC}^{\circ}(L)$ and $f \in \mathrm{LSC}^{-}(L)$. Then:

(1) $\operatorname{LSC}^{-}(L) \ni \bigvee\left(\downarrow_{\text {LSC }}(g)\right) \triangleleft g$.

(2) $f \triangleleft \wedge(\uparrow$ USC $(f)) \in \operatorname{USC}^{\circ}(L)$.

Proof: To prove (1), we first note that $h=\bigvee\left(\downarrow_{\text {LSC }}(g)\right)$ is a morphism from $\mathfrak{L}_{u}(\mathbb{R})$ into $L$ (Remarks $\left.3.3(1)\right)$. Also, $h \triangleleft g$ since

$$
g(-, r) \wedge \bigvee_{f \in \downarrow_{\mathrm{LSC}}(g)} f(r,-)=\bigvee_{f \in \downarrow_{\mathrm{LSC}}(g)}(g(-r) \wedge f(r,-))=0
$$

By Lemma 3.8, $h \in \operatorname{LSC}^{-}(L)$. Statement (2) is dual to (1).

Definition 4.2. Let

$$
(\cdot)^{\circ}: \operatorname{USC}^{\circ}(L) \rightarrow \operatorname{LSC}^{-}(L)
$$

and

$$
(\cdot)^{-}: \mathrm{LSC}^{-}(L) \rightarrow \mathrm{USC}^{\circ}(L)
$$

be defined by:

$$
g^{\circ}=\bigvee\left(\downarrow_{\mathrm{LSC}}(g)\right) \quad \text { and } \quad f^{-}=\bigwedge\left(\uparrow_{\mathrm{USC}}(f)\right) .
$$

Then $g^{\circ}$ is called the lower regularization of $g$ and $f^{-}$is called the upper regularization of $f$.

Note that, by Lemma $4.1,(\cdot)^{\circ}$ and $(\cdot)^{-}$are well-defined and $g^{\circ} \triangleleft g$ and $f \triangleleft$ $f^{-}$. Also, it is not difficult to check that $f^{-}=-(-f)^{\circ}$ for all $f \in \operatorname{LSC}^{-}(L)$.

Note. We have choosen to use $g^{\circ}$ and $f^{-}$rather than the classical notation $g_{*}$ and $f^{*}$ (see Discussion above) in order to avoid confusion with the well established notation $(\cdot)^{*}$ and $(\cdot)_{*}$ for morphisms in pointfree topology (cf. [8, page 40]). An advantage of our notation is that it emphasizes the parallelism between lower and upper regularizations and interior and closure operators in topology. E.g., $\left(1_{A}\right)^{\circ}=1_{A^{\circ}}$ and $\left(1_{A}\right)^{-}=1_{A^{-}}$.

Proposition 4.3. Let $g \in \mathrm{USC}^{\circ}(L)$ and $f \in \mathrm{LSC}^{-}(L)$. Then:

(1) $g^{\circ}(r,-)=\bigvee_{s>r} g(-, s)^{*}$.

(2) $f^{-}(-, r)=\bigvee_{s<r} f(s,-)^{*}$.

Proof: (1) We have shown within the proof of Lemma 3.8 that $h: \mathfrak{L}_{u}(\mathbb{R}) \rightarrow L$ defined by

$$
h(r,-)=\bigvee_{s>r} g(-, s)^{*}
$$


is proper l.s.c. and $h \triangleleft g$. By the definition of $g^{\circ}$ one has $h \leq g^{\circ}$. Since $g^{\circ} \triangleleft g$, we have

$$
g^{\circ}(r,-)=\bigvee_{s>r} g^{\circ}(s,-) \leq \bigvee_{s>r} g(-, s)^{*}=h(r,-) .
$$

Thus $g^{\circ} \leq h$ and $h=g^{\circ}$ follows.

(2) By (1) and the fact that $f^{-}=-(-f)^{\circ}$.

Remark 4.4. Notice that, classically, a pair $(f, g) \in \operatorname{LSC}(X, \mathbb{R}) \times \operatorname{USC}(X, \mathbb{R})$, in which $f$ minorizes and majorizes $g$, yields a continuous function $h=g=f$. In our pointfree setting the situation is more subtle. We have a bijection from $\mathrm{C}(L)$ to

$$
\mathcal{C}_{L}=\{(f, g) \in \operatorname{LSC}(L) \times \operatorname{USC}(L): f \triangleleft g \text { and } f \triangleright g\}
$$

given by

$$
\mathrm{C}(L) \ni h \mapsto\left(h_{\mid \mathfrak{L}_{u}(\mathbb{R})}, h_{\mid \mathfrak{L}_{l}(\mathbb{R})}\right) \in \mathcal{C}_{L} .
$$

The inverse of this bijection is given by $\mathcal{C}_{L} \ni(f, g) \longmapsto h \in \mathrm{C}(L)$ defined by

$$
h(p, q)=f(p,-) \wedge g(-, q) .
$$

Notation. In the latter case we shall write

$$
h=\langle f, g\rangle \text {. }
$$

In actual fact, elements of the pair $(f, g) \in \mathcal{C}_{L}$ uniquely determine each other as the following proposition shows.

Proposition 4.5. Let $f,-g \in \mathrm{LSC}(L)$. If $f$ minorizes and majorizes $g$, then $g=f^{-}$and $f=g^{\circ}$.

Proof: Clearly, $g(-, r)=\bigvee_{s<r} g(-, s) \leq \bigvee_{s<r} f(s,-)^{*}$. If $s<r$, then

$$
\begin{aligned}
f(s,-)^{*} & =f(s,-)^{*} \wedge 1 \\
& =f(s,-)^{*} \wedge(f(s,-) \vee g(-, r)) \\
& \leq g(-, r) .
\end{aligned}
$$

Hence $\bigvee_{s<r} f(s,-)^{*} \leq g(-, r)$. Thus $g=f^{-}$. Now, $f=g^{\circ}$ by what has just been proved and the fact that $-g$ minorizes and majorizes $-f$.

Proposition 4.6. Let $g \in \mathrm{USC}^{\circ}(L)$. Then the following hold:

(1) $g^{\circ-}(-, r)=\bigvee_{s<r} g(-, s)^{* *}$ for all $r \in \mathbb{Q}$.

(2) $g^{\circ-} \leq g$.

(3) If $g(-, r)^{*} \vee g(-, r)^{* *}=1$ for all $r \in \mathbb{Q}$, then $\left\langle g^{\circ}, g^{\circ-}\right\rangle \in \mathrm{C}(L)$. 
Proof: (1) For each $r \in \mathbb{Q}$ we have

$$
\begin{aligned}
g^{\circ-}(-, r) & =\bigvee_{s<r} g^{\circ}(s,-)^{*}=\bigvee_{s<r}\left(\bigvee_{t>s} g(-, t)^{*}\right)^{*} \\
& =\bigvee_{s<r} \bigwedge_{t>s} g(-, t)^{* *} \\
& \geq \bigvee_{s<r} g(-, s)^{* *},
\end{aligned}
$$

where the last inequality holds on account of $s \mapsto g(-, s)^{* *}$ being orderpreserving. On the other hand, $\bigwedge_{t>s} g(-, t)^{* *} \leq g(-, p)^{* *}$ whenever $s<p<$ $r$. Consequently, we get

$$
g^{\circ-}(-, r)=\bigvee_{s<r} \bigwedge_{t>s} g(-, t)^{* *} \leq \bigvee_{p<r} g(-, p)^{* *} .
$$

(2) By (1), we have $g^{\circ-}(-, r) \geq \bigvee_{s<r} g(-, s)=g(-, r)$, i.e. $g^{\circ-} \leq g$.

(3) Since $g^{\circ} \triangleleft g^{\circ-}$ always, it remains to show that $g^{\circ} g^{\circ-}$. Indeed, for each $p<s$ we have

$$
\begin{aligned}
g^{\circ}(p,-) \vee g^{\circ-}(-, s) & =\bigvee_{q>p} g(-, q)^{*} \vee \bigvee_{t<s} g(-, t)^{* *} \\
& \geq \bigvee_{p<r<s}\left(g(-, r)^{*} \vee g(-, r)^{* *}\right) \\
& =1
\end{aligned}
$$

Thus, $g^{\circ}$ majorizes $g^{\circ-}$.

The dual statement to Proposition 4.6 follows:

Proposition 4.7. Let $f \in \mathrm{LSC}^{-}(L)$. Then the following hold:

(1) $f^{-\circ}(r,-)=\bigvee_{s>r} f(s,-)^{* *}$ for all $r \in \mathbb{Q}$.

(2) $f \leq f^{-\circ}$.

(3) If $f(r,-)^{*} \vee f(r,-)^{* *}=1$ for all $r \in \mathbb{Q}$, then $\left\langle f^{-\circ}, f^{-}\right\rangle \in \mathrm{C}(L)$.

Definition $4.8([14])$. For each $a \in L$ we define the characteristic maps $u_{a} \in \mathrm{USC}^{\circ}(L)$ and $l_{a} \in \mathrm{LSC}^{-}(L)$ by

$$
u_{a}(-, q)=\left\{\begin{array}{ll}
0 & \text { if } q \leq 0, \\
a & \text { if } 0<q \leq 1, \\
1 & \text { if } q>1
\end{array} \quad \text { and } \quad l_{a}(p,-)= \begin{cases}1 & \text { if } p<0 \\
a & \text { if } 0 \leq p<1 \\
0 & \text { if } p \geq 1\end{cases}\right.
$$


Remark 4.9. One simply calculates that for each $a, b \in L$ the following hold:

(1) $\left(l_{a}\right)^{-}=u_{a^{*}}$ and $\left(u_{a}\right)^{\circ}=l_{a^{*}}$.

(2) $l_{a} \triangleleft u_{b}$ iff $a \wedge b=0$.

(3) $l_{a} \triangleright u_{b}$ iff $a \vee b=1$.

Corollary 4.10. $\left\langle l_{a}, u_{b}\right\rangle \in \mathrm{C}(L)$ if and only if $a$ and $b$ are complements of each other.

\section{Extremally disconnected frames}

A frame $L$ is called extremally disconnected if and only if $a^{*} \vee a^{* *}=1$ for all $a \in L$ (equivalently, if $a^{*}$ and $a^{* *}$ are complements of each other for every a).

Notation. When $f,-g \in \operatorname{Frm}\left(\mathfrak{L}_{u}(\mathbb{R}), L\right), f \triangleleft g$ and $h \in \mathrm{C}(L)$ is such that $f \leq h_{\mid \mathfrak{L}_{u}(\mathbb{R})}$ and $h_{\mid \mathfrak{L}_{l}(\mathbb{R})} \leq g$, we shall simply write

$$
f \leq h \leq g .
$$

Theorem 5.1. Let $L$ be a frame. The following are equivalent:

(1) $L$ is extremally disconnected.

(2) $\left\langle g^{\circ}, g^{-\circ}\right\rangle \in \mathrm{C}(L)$ for all $g \in \mathrm{USC}^{\circ}(L)$.

(3) $\left\langle f^{-\circ}, f^{-}\right\rangle \in \mathrm{C}(L)$ for all $f \in \mathrm{LSC}^{-}(L)$.

(4) If $f \in \mathrm{LSC}(L)$ minorizes $g \in \mathrm{USC}(L)$, then there exists an $h \in \mathrm{C}(L)$ such that $f \leq h \leq g$.

Proof: $(1) \Rightarrow(2) \Leftrightarrow(3)$ : By Proposition 4.6(3) we have the implication, while the equivalence follows by duality.

$(2) \Rightarrow(4)$ : By $f \triangleleft g, g$ is proper u.s.c. (Lemma 3.8). By hypothesis, $\left\langle g^{\circ}, g^{\circ-}\right\rangle$ is continuous and we claim $f \leq\left\langle g^{\circ}, g^{\circ-}\right\rangle \leq g$. Indeed, $f \leq g^{\circ}$ and, by Proposition $4.6(2), g^{\circ-} \leq g$.

$(4) \Rightarrow(1)$ : Let $a \in L$. By Remark $4.9(1), l_{a^{* *}} \triangleleft u_{a^{*}}$ and by hypothesis, there exists an $h=\langle f, g\rangle \in \mathrm{C}(L)$ such that $l_{a^{* *}} \leq h \leq u_{a^{*}}$. This means that $l_{a^{* *}} \leq f \triangleleft g$, hence $l_{a^{* *}} \triangleleft g$, i.e.,

$$
g\left(-, \frac{3}{4}\right) \leq l_{a^{* *}}\left(\frac{3}{4},-\right)^{*}=a^{* * *}=a^{*} .
$$

Similarly, $f \triangleleft g \leq u_{a^{*}}$, hence $f \triangleleft u_{a^{*}}$. This means that

$$
f\left(\frac{1}{4},-\right) \leq u_{a^{*}}\left(-, \frac{1}{4}\right)^{*}=a^{* *} .
$$


Since $f \triangleright g$, we conclude that

$$
1=g\left(-, \frac{3}{4}\right) \vee f\left(\frac{1}{4},-\right) \leq a^{*} \vee a^{* *} .
$$

This shows that $L$ is extremally disconnected.

Remarks 5.2. (1) It follows from Lemma 3.8 that in the assertion (4) of Theorem 5.1 one can equivalently replace the families $\operatorname{LSC}(L)$ and $\operatorname{USC}(L)$ by either $\operatorname{LSC}^{-}(L)$ and $\operatorname{USC}^{\circ}(L)$ or by $\operatorname{Frm}\left(\mathfrak{L}_{u}(\mathbb{R}), L\right)$ and $\operatorname{Frm}\left(\mathfrak{L}_{l}(\mathbb{R}), L\right)$.

(2) The equivalence between statements (1) and (4) (when phrased in terms of $\operatorname{Frm}\left(\mathfrak{L}_{u}(\mathbb{R}), L\right)$ and $\left.\operatorname{Frm}\left(\mathfrak{L}_{l}(\mathbb{R}), L\right)\right)$ is precisely [12, Theorem 2.2]. This explains the equivalence of insertion theorems mentioned in the Introduction. When applied to $\mathcal{O} X$ with $X$ an extremally disconnected space, Theorem 5.2 reduces to [10, Proposition and Corollary 1]. Historically, the topological version of statement (4) goes back to Stone [17] (see also [11]). Related literature include [5], [6] and [9].

Corollary 5.3 (Urysohn's type lemma). A frame $L$ is extremally disconnected if and only if, whenever $a \wedge b=0$ in $L$, there exists a continuous $h: \mathfrak{L}(\mathbb{R}) \rightarrow L$ such that $l_{a} \leq h \leq u_{b}$.

Proof: $\Rightarrow$ : By Corollary 4.10, $h=\left\langle l_{a^{* *}}, u_{a^{*}}\right\rangle \in \mathrm{C}(L)$. We claim $l_{a} \leq h \leq u_{b}$. Indeed, $l_{a} \leq l_{a^{* *}}$ and $u_{a^{*}} \leq u_{b}$ since $a \wedge b=0$.

$\Leftarrow$ : Let $a \in L$. By hypothesis, there exists an $h=\langle f, g\rangle \in \mathrm{C}(L)$ such that $l_{a^{* *}} \leq\langle f, g\rangle \leq u_{a^{*}}$ and we conclude that $L$ is extremally disconnected as in the proof of $(4) \Rightarrow(1)$ in Theorem 5.1.

Another corollary of Theorem 5.1 is a Tietze's type extension theorem characterizing extremally disconnected frames. Its topological version can be found in the book of Gillman and Jerison [3] where it is deduced from their Urysohn Extension Theorem. In a pointfree setting it appears in ([12, Theorem 3.1]), in the context of the already mentioned incorrect semicontinuity notions (see Introduction), as a consequence of the insertion theorem of [12], but in a very weak form: the authors of [12] merely work with what they call continuous chains, that is, frame homomorphisms with domain being generated by elements $(r,-)$ and $(-, r), r \in \mathbb{Q}$, subject to relations $\left(\mathrm{R} 1^{\prime}\right)-\left(\mathrm{R} 4^{\prime}\right)$. Below we provide a strengthened version of it for continuous bounded real functions. Our argument does not depend upon any insertion theorem. 
Let $S$ be a sublocale of $L$. One says that $\bar{h} \in \mathrm{C}(L)$ is a continuous extension of $h \in \mathrm{C}(S)$ if and only if the following diagram commutes

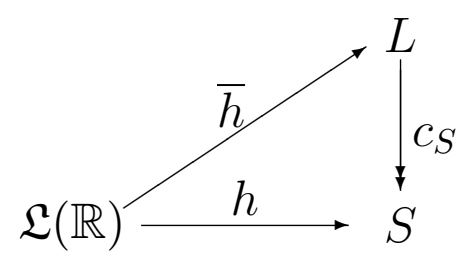

i.e. $c_{S} \circ \bar{h}=h$.

Remarks 5.4. (1) Let $a \in L$. If each $h \in \mathrm{C}(\downarrow a)$ extends continuously to $\bar{h} \in \mathrm{C}(L)$ (i.e., $c_{\downarrow a} \circ \bar{h}=h$ where $c_{\downarrow a}: L \rightarrow \downarrow a$ is defined by $\left.c_{\downarrow a}(x)=a \wedge x\right)$, then so does each member of $\mathrm{C}(\mathfrak{o}(a))$. Indeed, by Remark 2.1(1) there is a frame isomorphism $i: \mathfrak{o}(a) \rightarrow \downarrow a$ determined by $i(a \rightarrow x)=a \wedge x$. Thus, if $k \in \mathrm{C}(\mathfrak{o}(a))$, then there exists a $\bar{k} \in \mathrm{C}(L)$ such that $c_{\downarrow a} \circ \bar{k}=i \circ k$. Since $i \circ c_{\mathfrak{o}(a)}=c_{\downarrow a}$, it follows that $c_{\mathfrak{o}(a)} \circ \bar{k}=i^{-1} \circ i \circ k=k$.

(2) We will notationally distinguish the operations of taking pseudocomplements in $L$ and $\downarrow a$. The first is standardly denoted $(\cdot)^{*}$, while the second will be denoted by $(\cdot)\urcorner$. We note that $x\urcorner=a \wedge x^{*}$ for all $x \in \downarrow a$. Also $x\urcorner\urcorner=a \wedge x^{* *}$ whenever $x \in \downarrow a$. Indeed,

$$
x\urcorner\urcorner=a \wedge(x\urcorner)^{*}=a \wedge\left(a \wedge x^{*}\right)^{*} \geq a \wedge\left(a^{*} \vee x^{* *}\right)=a \wedge x^{* *} .
$$

Before continuing we observe that when $L$ is extremally disconnected, we already have the equality in the above calculation due to the dual De Morgan law which characterizes extremal disconnectedness (see [8, page 101]). For an arbitrary frame $L$ we continue as follows: since $a \wedge x^{*} \wedge\left(a \wedge x^{*}\right)^{*}=0$, it follows that $a \wedge\left(a \wedge x^{*}\right)^{*} \leq x^{* *}$ and so $\left.x\right\urcorner \leq a \wedge x^{* *}$. It may also be remarked that we have thus proved that: a frame $L$ is extremally disconnected if and only if $\downarrow a$ is extremally disconnected for any $a \in L$.

Recall that an $h \in \mathrm{C}(L)$ is said to be bounded if $h(p, q)=1$ for some $p<q$ (or, equivalently, if $h(-, p) \vee h(q,-)=0$ for some $p<q)$. In the sequel, $\mathrm{C}^{*}(L)$ stands for all the bounded members of $\mathrm{C}(L)$ such that $h(-, 0) \vee h(1,-)=0$.

Theorem 5.5. For $L$ a frame, the following are equivalent:

(1) $L$ is extremally disconnected.

(2) For every open sublocale $S$ of $L$, each $h \in \mathrm{C}^{*}(S)$ has a continuous extension $\bar{h} \in \mathrm{C}^{*}(L)$. 
Proof: $(1) \Rightarrow(2)$ : According to Remark $5.4(1)$, consider

$$
h=\left\langle h_{1}, h_{2}\right\rangle \in \mathrm{C}^{*}(\downarrow a) .
$$

We define $f: \mathfrak{L}_{u}(\mathbb{R}) \rightarrow L$ by

$$
f(r,-)= \begin{cases}h_{1}(r,-) & \text { if } r \geq 0, \\ 1 & \text { if } r<0 .\end{cases}
$$

It is easy to check that $f \in \mathrm{LSC}^{-}(L)$, for it minorizes a morphism $g: \mathfrak{L}_{l}(\mathbb{R}) \rightarrow$ $L$ given by $g(-, r)=h_{2}(-, r)$ if $r \leq 1$, and $g(-, r)=1$ otherwise. Indeed, since $h_{2}(-, 0)=0=h_{1}(1,-)$, we have

$$
f(r,-) \wedge g(-, r)= \begin{cases}g(-, r) \leq g(-, 0)=h_{2}(-, 0)=0 & \text { if } r \leq 0, \\ h_{1}(r,-) \wedge h_{2}(-, r)=0 & \text { if } 0<r<1 \\ f(r,-) \leq f(1,-)=h_{1}(1,-)=0 & \text { if } r \geq 1\end{cases}
$$

By the equivalence $(1) \Leftrightarrow(3)$ of Theorem $5.1, \bar{h}=\left\langle f^{-\circ}, f^{-}\right\rangle \in \mathrm{C}(L)$.

Claim 1: $\bar{h} \in \mathrm{C}^{*}(L)$. Indeed, $f^{-}(-, 0)=\bigvee_{s<0} f(s,-)^{* *}=0$ and

$$
f^{-\circ}(1,-)=\bigvee_{s>1} f(s,-)^{* *}=\bigvee_{s>1} h_{1}(s,-)^{* *}=0 .
$$

Claim 2: $c_{\downarrow a} \circ f^{-}=h_{2}$. Indeed, if $r \leq 0$, then

$$
c_{\downarrow a} \circ f^{-}(-, r)=a \wedge \bigvee_{s<r} f(s,-)^{*}=0=h_{2}(-, r) .
$$

With $r>0$ we have

$$
\begin{aligned}
c_{\downarrow a} \circ f^{-}(-, r) & =\bigvee_{s<r}\left(a \wedge f(s,-)^{*}\right) \\
& \left.=\bigvee_{s<r} h_{1}(s,-)\right\urcorner \\
& =h_{1}^{-}(-, r) \\
& =h_{2}(-, r),
\end{aligned}
$$

where $h_{1}^{-}=h_{2}$ by Proposition 4.5.

Claim 3: $c_{\downarrow a} \circ f^{-\circ}=h_{1}$. Indeed, if $r<0$, then

$$
c_{\downarrow a} \circ f^{-\circ}(r,-)=a \wedge \bigvee_{s>r} f(s,-)^{* *}=a \wedge 1=h_{1}(r,-)
$$


With $r \geq 0$ we have, by Remark 5.4(2),

$$
\begin{aligned}
c_{\downarrow a} \circ f^{-\circ}(r,-) & =a \wedge \bigvee_{s>r} f(s,-)^{* *} \\
& =\bigvee_{s>r}\left(a \wedge h_{1}(s,-)^{* *}\right) \\
& \left.\left.=\bigvee_{s>r} h_{1}(s,-)\right\urcorner\right\urcorner \\
& =h_{1}^{-\circ}(r,-) \\
& =h_{1}(r,-),
\end{aligned}
$$

since $h_{1}^{-\circ}=h_{1}$ by Proposition 4.5 again.

Finally,

$$
c_{\downarrow a} \circ \bar{h}=\left\langle c_{\downarrow a} \circ f^{-\circ}, c_{\downarrow a} \circ f^{-}\right\rangle=\left\langle h_{1}, h_{2}\right\rangle=h .
$$

$(2) \Rightarrow(1)$ : We have to show that $a^{*} \vee a^{* *}=1$ for all $a \in L$. For this purpose fix $a$ and consider the open sublocale $S=\mathfrak{o}\left(a \vee a^{*}\right)$. By Remark 2.1 (2) and Corollary 4.10,

$$
h=\left\langle l_{a^{*} \rightarrow a}, u_{a^{*}}\right\rangle \in \mathrm{C}^{*}(S) .
$$

By hypothesis, there exists an $\bar{h} \in \mathrm{C}^{*}(L)$ such that $c_{S} \circ \bar{h}=h$. For all $0<r<1$ we have

$$
\bar{h}(-, r) \leq\left(a \vee a^{*}\right) \rightarrow \bar{h}(-, r)=c_{S} \circ \bar{h}(-, r)=h(-, r)=a^{*} .
$$

By using (H1), we get

$$
\begin{aligned}
\bar{h}(-, r) & \geq\left(a \vee a^{*}\right) \wedge\left(\left(a \vee a^{*}\right) \rightarrow \bar{h}(-, r)\right) \\
& =\left(a \vee a^{*}\right) \wedge c_{S} \circ \bar{h}(-, r) \\
& =\left(a \vee a^{*}\right) \wedge a^{*}=a^{*},
\end{aligned}
$$

so that $\bar{h}(-, r)=a^{*}$. Since

$$
\bar{h}\left(-, \frac{1}{4}\right) \wedge \bar{h}\left(\frac{1}{4},-\right)=0,
$$

it follows that

$$
\bar{h}\left(\frac{1}{4},-\right) \leq \bar{h}\left(-, \frac{1}{4}\right)^{*}=a^{* *} .
$$

Finally,

$$
1=\bar{h}\left(-, \frac{3}{4}\right) \vee \bar{h}\left(\frac{1}{4},-\right) \leq a^{*} \vee a^{* *} .
$$




\section{References}

[1] B. Banaschewski, The Real Numbers in Pointfree Topology, Textos de Matemática, Série B, Vol. 12, Universidade de Coimbra, 1997.

[2] B. Banaschewski and C. J. Mulvey, Stone-Čech compactification of locales II, J. Pure Appl. Algebra 33 (1984) 107-122.

[3] L. Gillman and M. Jerison, Rings of Continuous Functions, Springer, New York, 1976.

[4] J. Gutiérrez García, T. Kubiak and J. Picado, Monotone insertion and monotone extension of frame homomorphisms, J. Pure Appl. Algebra (2007), (doi:10.1016/j.jpaa.2007.07.016).

[5] J. Gutiérrez García, T. Kubiak and M. A. de Prada Vicente, Insertion of lattice-valued and hedgehog-valued functions, Topology Appl. 153 (2006) 1458-1475.

[6] J. Gutiérrez García, T. Kubiak and M. A. de Prada Vicente, Generating and inserting continuous functions with values in bounded complete domains and hedgehog-like structures, Houston J. Math. (to appear).

[7] J. Gutiérrez García and J. Picado, On the algebraic representation of semicontinuity, J. Pure Appl. Algebra 210 (2007) 299-306.

[8] P. T. Johnstone, Stone Spaces, Cambridge Univ. Press, Cambridge, 1982.

[9] K. Klimorowski and T. Kubiak, Extending continuous L-real-valued functions from arbitrary subspaces, Fuzzy Sets Syst. 144 (2004) 395-401.

[10] T. Kubiak and M. A. de Prada Vicente, Hereditary normality plus extremal disconnectedness and insertion of continuous function, Math. Japonica 46 (1997) 403-405.

[11] E.P. Lane, A suficient condition for the insertion of a continuous function, Proc. Amer. Math. Soc. 49 (1975) 90-94.

[12] Y.-M. Li and Z.-H. Li, Constructive insertion theorems and extension theorems on extremally disconnected frames, Algebra Univers. 44 (2000) 271-281.

[13] Y.-M. Li and G.-J. Wang, Localic Katětov-Tong insertion theorem and localic Tietze extension theorem, Comment. Math. Univ. Carolinae 38 (1997) 801-814.

[14] J. Picado, A new look at localic interpolation theorems, Topology Appl. 153 (2006) 3203-3218.

[15] J. Picado and A. Pultr, Sublocale sets and sublocale lattices, Arch. Math. (Brno) 42 (2006) 409-418.

[16] J. Picado, A. Pultr and A. Tozzi, Locales in: M. C. Pedicchio and W. Tholen (Eds.), Categorical Foundations - Special Topics in Order, Algebra and Sheaf Theory, Encyclopedia of Mathematics and its Applications, Vol. 97, Cambridge Univ. Press, Cambridge, 2003, pp. 49-101.

[17] M.H. Stone, Boundedness properties in function-lattices, Canad. J. Math. 1 (1949) 176-186.

JaVier GutiÉRrez GarCía

Departamento de Matemáticas, Universidad del País Vasco-Euskal Herriko Unibertsitatea, Apartado 644, 48080, Bilbao, Spain

E-mail address: javier.gutierrezgarcia@ehu.es

TOMASZ KUBIAK

Wydziae Matematyki i Informatyki, Uniwersytet im. Adama Mickiewicza, Ul. UmultowsKA 87, 61-614 Poznań, Poland

E-mail address: tkubiak@amu.edu.pl

JoRge PiCADO

CMUC, Department of Mathematics, University of Coimbra, 3001-454 Coimbra, Portugal E-mail address: picado@mat.uc.pt 